\title{
Correlatos da atividade física em crianças e adolescentes: um estudo piloto
}

\author{
Physical activity correlates in children and adolescents: a pilot study \\ DOI: http://dx.doi.org/10.36453/2318-5104.2018.v16.n1.p217
}

\author{
Thiago Silva Piola ${ }^{1,2}$, Eliane Denise Araújo Bacil ${ }^{3}$, Priscila lumi Watanabe ${ }^{1}$, \\ Tiago Rocha Alves Costa ${ }^{1}$, Wagner Campos ${ }^{1}$ \\ ${ }^{1}$ Universidade Federal do Paraná (UFPR) \\ ${ }^{2}$ Centro Universitário Claretiano - Curitiba (CLARETIANO) \\ ${ }^{3}$ Universidade Positivo - Curitiba (UP)
}

\section{RESUMO}

Compreender os fatores associados (correlatos) ao comportamento sedentário de crianças e adolescentes é primordial para promover uma melhoria nas intervenções dos programas de atividade física. O objetivo do estudo foi verificar a associação entre a atividade física e seus correlatos em crianças e adolescentes. Foram analisados 444 escolares, sendo 214 meninos e 230 meninas com idades entre 8 a 18 anos de idade. As variáveis analisadas foram o nível socioeconômico, o estado nutricional, a maturação biológica, analisada pela maturação somática e sexual, o apoio social, a autoeficácia, o comportamento sedentário e o nível de atividade física. Nos resultados foram observadas associações entre a atividade física das meninas e o apoio social dos pais (OR: 0,12 IC95\%: 0,04-0,31), entre a atividade física dos meninos e o apoio social dos amigos (OR: 0,25 IC95\%: 0,09-0,67). Conclui-se que o apoio social está associado a prática de atividades físicas de meninos e meninas, devendo este correlato ser considerado em futuras intervenções.

PALAVRAS-CHAVE: Atividade Motora; Estilo de Vida Sedentário; Estado Nutricional; Apoio Social; Maturação Sexual; Autoeficácia.

\section{ABSTRACT}

Understanding the associated (correlated) factors to the sedentary behavior of children and adolescents is essential to promote an improvement in the interventions of physical activity programs. The aim of the study was to verify the association between physical activity and its correlates in children and adolescents. A total of 444 schoolchildren were analyzed, 214 boys and 230 girls aged 8 to 18 years. The analyzed variables were socioeconomic level, nutritional status, biological maturation, analyzed by somatic and sexual maturation, social support, self efficacy, sedentary behavior and level of physical activity. The results showed associations between the physical activity of the girls and the social support of the parents (OR: 0.12 CI95\%: 0.04-0.31), between the physical activity of the boys and the social support of the friends (OR: 0.25 IC95\%: 0.09-0.67). In conclusion, social support is associated with the practice of physical activities of boys and girls, and this correlate must be considered in future interventions.

KEYWORDS: Motor Activity; Sedentary Lifestyle; Nutritional Status; Social Support; Sexual Maturation; Self-Efficacy. 


\section{INTRODUÇÃO}

A prática regular de atividade física (AF) possibilita uma melhora em curto e longo prazo à saúde cardiovascular, óssea, muscular e psicológica pra crianças e adolescentes (GEBREMARIAM et al., 2012). Argumentos estes que mostram a importância de ser manter um estilo de vida ativo nesta fase, afinal, infância e adolescência correspondem a um período de grande vulnerabilidade e suscetibilidade de influências que colaboram na incorporação de comportamentos relacionados à saúde (VEIGA et al., 2009). Nesta perspectiva, compreender os fatores associados (correlatos) a prática habitual de AF é algo primordial para o desenvolvimento e a melhoria de intervenções na saúde pública (BAUMAN et al., 2012).

A AF é influenciada por correlatos biológicos, comportamentais, socioambientais e psicossociais (BAUMAN et al., 2012). O estado nutricional apresenta uma relação inversa com a prática de AF (CHUNG et al., 2012), a maturação biológica é um fator que pode alterar o padrão de AF de crianças e adolescentes (BACIL et al., 2015), o apoio social pode ser compreendido como qualquer comportamento que auxilia o indivíduo a alcançar metas ou resultados desejados e vem sendo citado como um importante correlato da AF (BURRUS et al., 2012), já a autoeficácia é a confiança na habilidade para realiza AF em situações específicas (BAUMAN et al., 2012) e o nível socioeconômico, que também apresenta uma relação com a AF (EIME et al., 2015).

O entendimento destes poderia contribuir, por exemplo, para uma redução do abandono das práticas esportiva, a qual no sul do Brasil é deixada de lado por 30,4\% dos adolescentes até os 15 anos de idade e 41,8\% entre os jovens de 16 a 24 anos de idade (BRASIL, 2015). Assim sendo, o objetivo deste estudo foi verificar a associação entre a AF e as seguintes variáveis: estado nutricional, maturação biológica, comportamento sedentário, apoio social e autoeficácia.

\section{MÉTODOS}

Os participantes do estudo foram 444 escolares escolhidos por conveniência, sendo 214 meninos $(12,98 \pm 2,91$ anos) e 230 meninas (12,99 $\pm 2,66$ anos) com idade média de $13,40 \pm 2,77$ de duas escolas públicas da cidade de Curitiba (PR). A coleta de dados foi efetuada de novembro de 2013 a fevereiro de 2014 por uma equipe treinada do Centro de Estudos em Atividade Física e Saúde (CEAFS-UFPR). Foram considerados elegíveis os alunos que estavam na sala de aula no dia da coleta de dados, que tinham entre oito a 18,9 anos de idade e que não apresentavam nenhuma limitação física ou mental que limite ou impeça a prática de atividades físicas.

Inicialmente foi solicitada autorização das escolas para a realização do estudo e agendado os dias de coleta de dados. Uma visita foi marcada à escola para apresentação dos objetivos e relevância do estudo, bem como foi realizado um treinamento com a equipe para melhorar a confiabilidade da coleta de dados. No dia anterior a coleta de dados foi entregue aos alunos o Termo de Consentimento Livre e Esclarecido (TCLE) para os pais ou responsáveis autorizarem seus filhos a participarem do estudo. No dia estipulado da coleta de dados, de posse deste documento, os escolares responderam o questionário em sala de aula, durante o horário normal de aula, a partir de orientações previas do grupo de aplicação e posteriormente realizaram a avaliação antropométrica.

A determinação do nível socioeconômico foi realizada através do Critério de Classificação Econômica Brasil - CCEB (ABEP, 2013), o qual contabiliza a quantidade de itens existentes na casa de cada aluno. Os alunos foram instruídos a responder se apresentavam em seu domicílio cada item da lista e a sua quantidade, o grau de instrução do chefe da família ou do responsável que o sustenta e a presença de serviço público (água encanada e rua pavimentada). Para fins de análise os escolares foram agrupados nas classes econômicas A/B e C. Não havia escolares de classes econômicas D e E. O grau de escolaridade do pai e da mãe foi classificado em fundamental incompleto, fundamental completo, médio incompleto, médio completo, superior incompleto, superior completo.

O estado nutricional foi avaliado pelo Índice de Massa Corporal $\left(\mathrm{Kg} / \mathrm{m}^{2}\right)$, a partir das medidas de massa corporal (Kg) e estatura (m), seguindo os procedimentos propostos por Alvarez, Pavan e Petroski (2003) e classificado por Cole et al. (2000) ajustado para idade e sexo. Os escolares foram classificados como "baixo peso/normal", "sobrepeso" e "obesidade". 
A maturação biológica foi avaliada pela análise da maturação somática e maturação sexual. A maturação somática foi avaliada pela Idade do Pico de Velocidade de Altura (MIRWALD et al., 2002). Para tanto foram utilizadas as medidas de estatura, altura sentada, comprimento da perna, massa corporal e idade cronológica. A idade cronológica foi coletada em idade decimal. A medida de altura sentado seguiu os procedimentos descritos por Alvarez, Pavan e Petroski (2003). O comprimento da perna foi obtido a partir da diferença entre a estatura e a altura sentado. A equação preditiva para estimar a idade do pico de velocidade de altura (IPVA) é um método não invasivo, viável e fornece um indicador de timing entre os indivíduos para permitir comparações entre grupos de maturidade biológica. Segundo Mirwald et al. (2002) o coeficiente de validade entre a idade esquelética e o desvio de maturidade é de 0,83 sugerindo resultado aceitável para estimar o estado de maturidade. Os escolares foram classificados em: maturados precoce, no tempo e tardio. A maturação sexual foi determinada pelos estágios de Tanner (TANNER, 1962) através do método de autoavaliação da pilosidade pubiana, em um espaço reservado e privativo. Os estágios maturacionais foram classificados em escala de 1 a 5, sendo que o estágio 1 equivale ao nível "pré-púbere", os estágios intermediários 2, 3 e 4 ao nível "púbere" e o estágio 5 ao nível "pós-púbere". Este método foi determinado por autoavaliação comparativa com pranchas ilustrativas do aparecimento de pelos pubianos tanto em meninos quanto em meninas.

Os fatores psicossociais mensurados foram apoio social dos pais e dos amigos para prática de AF e percepção de autoeficácia. $\mathrm{O}$ apoio social para a prática de atividade física foi avaliado por uma escala com doze itens, seis em cada grupo (pais e amigos). Os adolescentes reportaram a frequência (nunca, raramente, frequentemente, sempre) com que pais e amigos forneceram algum tipo de apoio social (estimularam, praticaram juntos, assistiram, convidaram, comentaram sobre a prática da atividade física, forneceram transporte) durante uma típica semana (FARIAS JÚNIOR et al., 2011).

A autoeficácia foi avaliada pela escala Likert de quatro pontos composta de 12 itens, sendo: 1 (discordo muito), 2 (discordo), 3 (concordo) e 4 (concordo muito) (FARIAS JÚNIOR et al., 2011). Foram construídos escores para cada um dos fatores psicossociais a partir do somatório das respostas de cada item das respectivas escalas. Escores elevados indicam aspectos mais favoráveis a prática de AF. Como não há classificação para os resultados dos escores dessas variáveis para fins de análise foram calculados tercis separados por sexo para cada um dos fatores. Sendo assim o primeiro tercil e o último tercil de cada variável indicam respectivamente os piores e os melhores resultados.

A atividade física foi mensurada por um questionário (adaptação do Self-administered Physical Activity Checklist) (FARIAS JÚNIOR et al., 2012), o qual possui questões sobre a frequência (dias da semana) e duração (horas e minutos por dia) da participação em 24 atividades físicas de intensidade moderada a vigorosa, com a possibilidade do adolescente acrescentar mais duas atividades. Para responder o questionário foi considerada a semana anterior a da coleta de dados. Para a obtenção do nível de atividade física determinou-se o somatório do produto do tempo dispendido em cada uma das atividades físicas pelas respectivas frequências de prática, obtendo-se o nível de atividade física em minutos por semana. Foram considerados suficientemente ativos os escolares que praticaram atividades físicas moderadas ou vigorosas por um tempo igual ou maior do que 420 minutos/semana e insuficientemente ativos aqueles que se exercitaram por um tempo menor (WHO, 2010).

O comportamento sedentário foi obtido pelo Questionário de Atividades Sedentárias em Adolescentes Brasileiros - QASA (GUIMARÃES et al., 2013), composto por 13 itens, no qual os estudantes relatam o tempo gasto em atividades sedentárias em horas e/ou minutos durante cada dia de semana e durante um típico final de semana. Para fins de resultado final, efetuou-se o cálculo de média ponderada adotando-se o seguinte procedimento matemático: tempo dos dias de semana multiplicado por cinco, somado ao tempo dos dias de fim de semana multiplicado por dois. Este resultado foi dividido por sete para se obter o número médio de horas por dia de comportamento sedentário. Para fins de análise foram calculados tercis separados por sexo para cada um dos fatores. Sendo assim o primeiro e o último tercil de cada variável indicam respectivamente os piores e os melhores resultados.

O cálculo amostral a posteriori, realizado no GPower, versão 3.1.9.2, para verificar o poder amostral, indicou que 214 meninos poderiam identificar razões de chances acima de 2,20 como risco e abaixo 0,42 de como proteção. E para 230 meninas foi possível estimar razões de chances acima de 2,15 como risco e de 0,47 como proteção.

Para a descrição das variáveis, estratificadas por sexo, utilizou-se a distribuição de frequências absolutas 
e relativas e o teste do qui-quadrado para proporções. A regressão logística binária foi utilizada para avaliar a associação bruta e ajustada entre atividade física (suficientemente ativo=0 e insuficientemente ativo=1) e as variáveis estado nutricional, maturação somática, maturação sexual, comportamento sedentário, apoio social de pais e amigos e autoeficácia. Na análise bruta cada variável independente foi incluída separadamente e na análise ajustada todas as variáveis independentes e confundidoras foram incluídas. Os fatores de confusão considerados neste estudo foram idade, nível socioeconômico e escolaridade do pai e da mãe. A variáveis estado nutricional, maturação somática, maturação sexual, comportamento sedentário, apoio social dos pais, apoio social dos amigos e a autoeficácia foram analisadas no modelo ajustado, permanecendo aquelas com valor $p<0,20$. Finalmente um termo de interação foi incluído no modelo ajustado para verificar o papel moderador da idade e nível socioeconômico na associação entre as variáveis independentes e dependente.

As análises estatísticas foram efetuadas no SPSS, v. 24, adotando-se um nível de significância de $5 \%$. 0 estudo foi aprovado pelo Comitê de Ética em Pesquisa com Seres Humanos da Universidade Federal do Paraná (Parecer $n^{\circ}$. 896.737; CAAE: 36759414.0.0000.0102), de acordo com a Resolução 466/2012 do Conselho Nacional de Saúde.

\section{RESULTADOS E DISCUSSÃO}

A Tabela 1 apresenta a distribuição da amostra de acordo com as variáveis do estudo, segundo a estratificação por sexo. Não foram observadas diferenças significativas entre o estado nutricional, classe econômica, escolaridade dos pais, maturação somática e sexual, bem como entre a autoeficácia. No entanto, o nível de atividade física, comportamento sedentário e apoio social dos pais e amigos diferiram entre meninos e meninas $(p \leq 0,05)$.

Conforme demonstrado pelos resultados da regressão logística, expostos na Tabela 2, apenas na análise bruta os indivíduos obesos apresentaram maiores chances de possuírem níveis insuficientes de atividade física em comparação aos eutrófico, após o ajuste esta associação perdeu significância para ambos os sexos. Tanto a maturação somática quanto a sexual, assim como o comportamento sedentário e a autoeficácia não apresentaram associações significativas com a prática insuficiente de atividade física, após o ajuste. Observouse que meninas pertencentes ao 3 o tercil de apoio social dos pais apresentaram 0,12 vezes menos chances de serem insuficientemente ativas em comparação as do 1 o tercil (IC95\% 0,04-0,31). Já os meninos apresentaram associação entre a AF insuficiente e o apoio social dos amigos (OR: 0,25; IC95\% 0,09-0,67).

No presente estudo, não houve associação entre o estado nutricional e o nível de atividade física dos participantes. Em contraste, outros estudos verificaram uma associação inversa significativa entre o excesso de peso e o nível de atividade física, tanto em crianças, quanto em adolescentes (MUTHURI et al., 2014; SOUZA et al., 2010). Esta relação pode ocorrer devido à contribuição da falta de atividade física para o aumento do excesso de peso como pela tendência de indivíduos com sobrepeso evitarem a prática de atividade física (GUILHERME et al., 2015).

Em relação à maturação somática e sexual, não foram observadas associações significativas com a prática insuficiente de atividade física. Estudos sugerem que, especialmente em meninas, o avanço dos estágios maturacionais está relacionado com o declínio dos níveis de atividade física, independente da idade cronológica (CUMMING et al., 2012). Tal relação pode ser decorrente do impacto das transformações fisiológicas, psicológicas e sociais que são características deste período de transição (SHERAR et al., 2010). Entretanto ainda há uma inconsistência acerca do tema.

Sabe-se que os comportamentos de risco à saúde, tendem a agregar-se potencializando o risco do desenvolvimento de doenças cardiovasculares (FUCHS et al., 2008). Além disto, evidências apontam que um indivíduo pode manter um elevado tempo gasto em comportamentos sedentários, mas mesmo assim apresentar elevados níveis de atividade física e que o oposto também é verdadeiro (LEECH et al., 2014). Isto pode esclarecer a falta de relação entre o comportamento sedentário e a prática insuficiente de atividade física, na presente pesquisa.

Além do estado nutricional e da maturação, outro fator que pode influenciar a prática de atividade física é o apoio social. Na amostra avaliada verificou-se que o grupo de meninas do 3o tercil de apoio social dos pais apresentou 0,12 vezes menos chances de ser insuficientemente ativo em comparação ao 10 tercil. Já para os meninos foi o apoio social dos amigos que conferiu ao grupo uma menor chance de ser insuficientemente ativo (OR:0,25; IC95\%0,09-0,67). 
Tabela 1. Distribuição dos participantes do estudo, total e estratificado por sexo, segundo variáveis selecionadas.

\begin{tabular}{|c|c|c|c|c|c|c|c|}
\hline \multirow{2}{*}{ Variável } & \multicolumn{2}{|c|}{ Total } & \multicolumn{2}{|c|}{ Masculino } & \multicolumn{2}{|c|}{ Feminino } & \multirow{2}{*}{$\mathbf{p}$} \\
\hline & $\mathbf{n}$ & $\%$ & $\mathbf{n}$ & $\%$ & $\mathbf{N}$ & $\%$ & \\
\hline \multicolumn{8}{|l|}{ Sexo } \\
\hline Masculino & 214 & 48,2 & & & & & \\
\hline Feminino & 230 & 51,8 & & & & & \\
\hline \multicolumn{8}{|l|}{ Estado nutricional } \\
\hline Eutrófico & 279 & 72,3 & 133 & 71,1 & 146 & 73,4 & \multirow{3}{*}{0,79} \\
\hline Sobrepeso & 71 & 18,4 & 37 & 19,8 & 34 & 17,1 & \\
\hline Obeso & 36 & 9,3 & 17 & 9,1 & 19 & 9,5 & \\
\hline \multicolumn{8}{|l|}{ NSE } \\
\hline NSE A/B & 294 & 79,7 & 135 & 78,5 & 159 & 80,7 & \multirow{2}{*}{0,34} \\
\hline NSE C & 75 & 20,3 & 37 & 21,5 & 38 & 19,3 & \\
\hline \multicolumn{8}{|l|}{ Escolaridade do pai } \\
\hline$<8$ anos de estudo & 122 & 34,4 & 57 & 34,5 & 65 & 34,2 & \multirow{2}{*}{0,52} \\
\hline$>8$ anos de estudo & 233 & 65,6 & 108 & 65,5 & 125 & 65,8 & \\
\hline \multicolumn{8}{|l|}{ Escolaridade da mãe } \\
\hline$<8$ anos de estudo & 126 & 33,1 & 57 & 31,8 & 69 & 34,2 & \multirow{2}{*}{0,36} \\
\hline$>8$ anos de estudo & 255 & 66,9 & 122 & 68,2 & 133 & 65,8 & \\
\hline \multicolumn{8}{|l|}{ Maturação somática } \\
\hline Maturação no tempo & 256 & 66,1 & 124 & 66,3 & 132 & 66,0 & \multirow{3}{*}{0,98} \\
\hline Maturação precoce & 63 & 16,3 & 30 & 16,0 & 33 & 16,5 & \\
\hline Maturação tardia & 68 & 17,6 & 33 & 17,6 & 35 & 17,5 & \\
\hline \multicolumn{8}{|l|}{ Maturação sexual } \\
\hline Púbere & 279 & 71,9 & 134 & 71,7 & 145 & 72,1 & \multirow{3}{*}{0,73} \\
\hline Pre-pubere & 57 & 14,7 & 26 & 13,9 & 31 & 15,4 & \\
\hline Pos-pubere & 52 & 13,4 & 27 & 14,4 & 25 & 12,4 & \\
\hline \multicolumn{8}{|l|}{ Atividade Física } \\
\hline Ativo & 282 & 63,5 & 149 & 69,6 & 133 & 57,8 & \multirow{2}{*}{0,01} \\
\hline Insuficientemente ativo & 162 & 36,5 & 65 & 30,4 & 97 & 42,2 & \\
\hline \multicolumn{8}{|l|}{ Comportamento sedentário } \\
\hline 10 tercil & 148 & 33,4 & 89 & 41,8 & 59 & 25,7 & \multirow{3}{*}{0,01} \\
\hline 20 tercil & 147 & 33,2 & 70 & 32,9 & 77 & 33,5 & \\
\hline 3 tercil & 148 & 33,4 & 54 & 25,4 & 94 & 40,9 & \\
\hline \multicolumn{8}{|l|}{ Apoio social dos pais } \\
\hline 10 tercil & 123 & 27,7 & 50 & 23,4 & 73 & 31,7 & \\
\hline 20 tercil & 160 & 36,0 & 79 & 36,9 & 81 & 35,2 & 0,04 \\
\hline 3o tercil & 161 & 36,3 & 85 & 39,7 & 76 & 33,0 & \\
\hline Apoio social dos amigos & & & & & & & \\
\hline 10 tercil & 125 & 28,2 & 50 & 23,4 & 75 & 32,6 & \\
\hline 20 tercil & 167 & 37,6 & 74 & 34,6 & 93 & 40,4 & 0,01 \\
\hline 30 tercil & 152 & 34,2 & 90 & 42,1 & 62 & 27,0 & \\
\hline Autoeficácia & & & & & & & \\
\hline 10 tercil & 121 & 27,3 & 69 & 32,2 & 52 & 22,6 & \\
\hline 29 tercil & 143 & 32,2 & 58 & 27,1 & 85 & 37,0 & 0,22 \\
\hline 3ㅇ tercil & 180 & 40,5 & 87 & 40,7 & 93 & 40,4 & \\
\hline
\end{tabular}


Em uma amostra representativa de adolescentes brasileiros, verificou-se que tanto o apoio dos amigos quanto da família, em especial a companhia, estava associado com a prática de atividade física, em ambos os sexos (PRADO et al., 2014). Segundo Seabra et al. (2008), durante a infância os pais exercem grande influência sobre o comportamento dos filhos, mas com o avanço da idade os hábitos e comportamentos dos pares se tornam mais relevantes. No entanto, há indícios de que, apesar do declínio de apoio social dos pais com o decorrer do tempo, ter recebido o suporte dos pais para praticar atividade física foi um importante fator para manutenção da atividade física durante a adolescência, em meninas (DAVISON; JAGO, 2009).

Tabela 2. Associação entre atividade física insuficiente e estado nutricional, maturação biológica, comportamento sedentário, apoio social e autoeficácia, Curitiba, 2014.

\begin{tabular}{|c|c|c|c|c|}
\hline \multirow{3}{*}{ Variável } & \multicolumn{2}{|c|}{ Masculino } & \multicolumn{2}{|c|}{ Feminino } \\
\hline & $\begin{array}{c}\text { Análise } \\
\text { bruta }\end{array}$ & $\begin{array}{c}\text { Análise } \\
\text { ajustada }\end{array}$ & $\begin{array}{c}\text { Análise } \\
\text { bruta }\end{array}$ & $\begin{array}{c}\text { Análise } \\
\text { ajustada }\end{array}$ \\
\hline & OR (IC95\%) & OR (IC95\%) & OR (IC95\%) & OR (IC95\%) \\
\hline \multicolumn{5}{|l|}{ Estado nutricional } \\
\hline Eutrófico & 1,00 & 1,00 & 1,00 & 1,00 \\
\hline Sobrepeso & $0,80(0,35-1,86)$ & $0,86(0,32-2,36)$ & $0,59(0,27-1,28)$ & $0,64(0,25-1,66)$ \\
\hline Obeso & $2,81(1,01-7,83)$ & $1,92(0,48-7,65)$ & $0,29(0,09-0,91)$ & $0,63(0,17-2,42)$ \\
\hline \multicolumn{5}{|l|}{ Maturação somática } \\
\hline Maturação no tempo & 1,00 & 1,00 & 1,00 & 1,00 \\
\hline Maturação precoce & $1,87(0,82-4,24)$ & $1,02(0,32-3,26)$ & $0,54(0,24-1,22)$ & $0,63(0,19-2,02)$ \\
\hline Maturação tardia & $0,66(0,26-1,65)$ & $0,93(0,33-2,63)$ & $1,17(0,55-2,46)$ & $1,29(0,53-3,19)$ \\
\hline \multicolumn{5}{|l|}{ Maturação sexual } \\
\hline Púbere & 1,00 & 1,00 & 1,00 & 1,00 \\
\hline Pre-pubere & $3,06(1,30-7,22)$ & $1,97(0,52-7,52)$ & $1,29(0,59-2,81)$ & $0,64(0,19-2,14)$ \\
\hline Pos-pubere & $0,60(0,21-1,69)$ & $0,41(0,11-1,49)$ & $0,92(0,39-2,18)$ & $0,51(0,17-1,55)$ \\
\hline \multicolumn{5}{|l|}{ Comportamento sedentário } \\
\hline 10 tercil & 1,00 & 1,00 & 1,00 & 1,00 \\
\hline 20 tercil & $0,95(0,48-1,86)$ & $0,90(0,40-2,06)$ & $0,79(0,40-1,56)$ & $1,11(0,48-2,56)$ \\
\hline 3o tercil & $0,72(0,34-1,54)$ & $0,95(0,39-2,30)$ & $0,72(0,37-1,39)$ & $0,85(0,38-1,93)$ \\
\hline \multicolumn{5}{|l|}{ Apoio social dos pais } \\
\hline 10 tercil & 1,00 & 1,00 & 1,00 & 1,00 \\
\hline 2o tercil & $0,52(0,25-1,09)$ & $0,67(0,27-1,68)$ & $0,51(0,27-0,98)$ & $0,56(0,26-1,20)$ \\
\hline 30 tercil & $0,39(0,18-0,83)$ & $0,48(0,19-1,23)$ & $0,09(0,04-0,21)$ & $0,12(0,04-0,31)$ \\
\hline \multicolumn{5}{|l|}{ Apoio social dos amigos } \\
\hline 10 tercil & 1,00 & 1,00 & 1,00 & 1,00 \\
\hline $2 \circ$ tercil & $0,67(0,32-1,40)$ & $0,86(0,36-2,08)$ & $0,36(0,19-0,67)$ & $0,67(0,32-1,41)$ \\
\hline 3o tercil & $0,23(0,11-0,51)$ & $0,25(0,09-0,67)$ & $0,19(0,09-0,40)$ & $0,44(0,17-1,14)$ \\
\hline \multicolumn{5}{|l|}{ Autoeficácia } \\
\hline 10 tercil & 1,00 & 1,00 & 1,00 & 1,00 \\
\hline 20 tercil & $1,06(0,51-2,20)$ & $1,18(0,51-2,72)$ & $0,64(0,31-1,29)$ & $0,60(0,24-1,49)$ \\
\hline 3o tercil & $0,56(0,28-1,13)$ & $0,50(0,20-1,23)$ & $1,00(0,51-1,98)$ & $0,94(0,39-2,25)$ \\
\hline
\end{tabular}

Neste estudo a autoeficácia não esteve associada à atividade física insuficiente, concordando com achados de Matias et al. (2009), que não observaram diferenças ao comparar a autoeficácia entre os grupos ativo e sedentário. Tais achados contrastam com os de Souza et al. (2013), que encontraram uma relação positiva entre a autoeficácia e a atividade física em adolescentes de Curitiba (PR). De maneira semelhante, em estudo realizado com meninas adolescentes, o apoio social esteve relacionado indiretamente com o aumento 
da atividade física auto reportada por meio da autoeficácia (MOTL et al., 2007).

Como limitações deste estudo destacam-se a avaliação da atividade física por meio de questionário auto reportado e a utilização de uma amostra não probabilística, o que dificulta extrapolação dos resultados. Sugerese que futuros estudos contemplem a avaliação da atividade física de forma direta (acelerometria), bem como tenham um caráter longitudinal para uma melhor análise das relações causais, além de que intervenções para esta faixa etária deveriam considerar o apoio social de pais e amigos.

\section{CONCLUSÃO}

Por fim, os achados do presente estudo sugerem que o apoio social está associado a alterações no nível de atividade física de crianças e adolescentes. Recomenda-se a investigação da associação da AF e seus correlatos com amostras probabilísticas, as quais não estão presentes neste artigo por se tratar de um estudo piloto. As variáveis psicossociais (apoio social e autoeficácia) tem sua associação com a AF pouco exploradas pela literatura, deixando esta lacuna como sugestão para futuras investigações.

\section{REFERÊNCIAS}

ABEP. Critério de Classificação Econômica Brasil. Associação Brasileira de Empresas de Pesquisa, v. 2013, 2013. Disponível em: <http://www.abep.org/codigos-e-guias-da-abep>. Acessado em: 01 de fevereiro de 2014.

ALVAREZ, B. R.; PAVAN, A. L.; PETROSKI, E. Alturas e comprimentos. In: ALVAREZ, B. R.; PAVAN, A. L.; PETROSKI, E. Antropometria: Técnicas e Mensurações. 2. ed. Porto Alegre: Pallotti, 2003. p. 31-45.

BACIL, E. D.; MAZZARDO JUNIOR, O.; RECH, C. R.; LEGNANI, R. F.; CAMPOS, W. Physical activity and biological maturation: a systematic review. Revista Paulista de Pediatria, São Paulo, v. 33, n. 1, p. 114-21, 2015.

BAUMAN, A. E.; REIS, R. S.; SALLIS, J. F.; WELLS, J. C.; LOOS, R. J.; MARTIN, B. W. Correlates of physical activity: why are some people physically active and others not? The Lancet, London, v. 380, n. 9838, p. 258-71, 2012.

BRASIL. Diagnóstico nacional do esporte. Ministério do esporte, 2015. Disponível em: < $\underline{\text { http://www. }}$ esporte.gov.br/diesporte/>. Acessado em: 01 de junho de 2016.

BURRUS, B.; LEEKS, K. D.; SIPE, T. A.; DOLINA, S.; SOLER, R.; ELDER, R.; BARRIOS, L.; GREENSPAN, A.; FISHBEIN, D.; LINDEGREN, M. L.; ACHREKAR, A.; DITTUS, P. Person-to-person interventions targeted to parents and other caregivers to improve adolescent health: a community guide systematic review. American Journal of Prevention Medicine, Washington, v. 42, n. 3, p. 316-26, 2012.

CHUNG, A. E.; SKINNER, A. C.; STEINER, M. J.; PERRIN, E. M. Physical activity and BMI in a nationally representative sample of children and adolescents. Clinical Pediatrics, Philadelphy, v. 51, n. 2, p. 122-9, 2012.

COLE, T. J.; BELLIZZI, M. C.; FLEGAL, K. M.; DIETZ, W. H. Establishing a standard definition for child overweight and obesity worldwide: international survey. British Medical Journal, London, v. 320, n. 1240, p. 1-6, 2000.

CUMMING, S. P.; SHERAR, L. B.; PINDUS, D. M.; COELHO-E-SILVA, M. J.; MALINA, R. M.; JARDINE, P. R. A biocultural model of maturity-associated variance in adolescent physical activity. International Review of Sport \& Exercise Psychology, London, v. 5, n. 1, p. 23-43, 2012.

DAVISON, K. K.; JAGO, R. Change in parent and peer support across ages 9 to $15 \mathrm{yr}$ and adolescent girls' physical activity. Medicine \& Science in Sports \& Exercise, Indianapolis, v. 41, n. 9, p. 1816-25, 2009.

EIME, R. M.; CHARITY, M. J.; HARVEY, J. T.; PAYNE, W. R. Participation in sport and physical activity: associations with socio-economic status and geographical remoteness. BMC Public Health, London, v. 15, n. 1, p. 1, 2015. 
FARIAS JÚNIOR, J. C.; LOPES, A. S.; MOTA, J.; SANTOS, M. P.; RIBEIRO, J. C.; HALLAL, P. C. Validade e reprodutibilidade de um questionário para medida de atividade física em adolescentes: uma adaptação do self-administered physical activity checklist. Revista Brasileira de Epidemiologia, Rio de Janeiro, v. 15, n. 1, p. 13, 2012.

FARIAS JÚNIOR, J. C.; LOPES, A. S.; REIS, R. S.; NASCIMENTO, J. V.; BORGATTO, A. F.; HALLAL, P. C. Development and validation of a questionnaire measuring factors associated with physical activity in adolescents. Revista Brasileira de Saúde Materno Infantil, Recife, v. 11, n. 3, p. 301-12, 2011.

FUCHS, S. C.; MOREIRA, L. B.; CAMEY, S. A.; MOREIRA, M. B.; FUCHS, F. D. Clustering of risk factors for cardiovascular disease among women in southern Brazil: a population-based study. Caderno de Saúde Pública, Rio de Janeiro, v. 24, Suppl. 2, p. s285-s293, 2008.

GEBREMARIAM, M. K.; I, H. B.; L, F. A.; OMMUNDSEN, Y.; BJELLAND, M.; LIEN, N. Stability and change in potential correlates of physical activity and association with pubertal status among Norwegian children in the transition between childhood and adolescence. International Journal of Behavioral Nutrition and Physical Activity, London, v. 9, n. 56, p. 56, 2012.

GUILHERME, F. R.; MOLENA-FERNANDES, C. A.; GUILHERME, V. R.; FAVERO, M. T.; REIS, E. J.; RINALDI, W. Physical inactivity and anthropometric measures in school children from Paranavai, Parana, Brazil. Revista Paulista de Pediatria, São Paulo, v. 33, n. 1, p. 50-5, 2015.

GUIMARÃES, R. F.; SILVA, M. P.; LEGNANI, E.; MAZZARDO, O.; CAMPOS, W. Reproducibility of adolescent sedentary activity questionnaire (ASAQ) in Brazilian adolescents. Revista Brasileira de Cineantropometria \& Desempenho Humano, Florianópolis, v. 15, n. 3, p. 276-25, 2013.

LEECH, R. M.; MCNAUGHTON, S. A.; TIMPERIO, A. The clustering of diet, physical activity and sedentary behavior in children and adolescents: a review. International Journal of Behavioral Nutrition and Physical Activity, London, v. 11, n. 4, p. 1-9, 2014.

MATIAS, T. S.; ROLIM, M. K. S. B.; KRETZER, F. L.; SCHMOELZ, C. P.; VASCONCELLOS, D. I. C.; ANDRADE, A. Estilo de vida, nível habitual de atividade física e percepção de auto-eficácia de adolescentes. Revista da Educação Física/UEM, Maringá, v. 20, n. 2, p. 235-43, 2009.

MIRWALD, R. L.; BAXTER-JONES, A. D.; BAILEY, D. A.; BEUNEN, G. P. An assessment of maturity from anthropometric measurements. Medicine and Science in Sports Exercise, Indianapolis, v. 34, n. 4, p. 689-94, 2002

MOTL, R. W.; DISHMAN, R. K.; SAUNDERS, R. P.; DOWDA, M.; PATE, R. R. Perceptions of physical and social environment variables and self-efficacy as correlates of self-reported physical activity among adolescent girls. Journal of Pediatric Psychology, Lawrence, v. 32, n. 1, p. 6-12, 2007.

MUTHURI, S. K.; WACHIRA, L. J. M.; ONYWERA, V. O.; TREMBLAY, M. S. Correlates of objectively measured overweight/obesity and physical activity in Kenyan school children: results from ISCOLEKenya. BMC Public Health, London, v. 14, n. 436, p. 1-11, 2014.

PRADO, C. V.; LIMA, A. V.; FERMINO, R. C.; ANEZ, C. R. R.; REIS, R. S. Social support and physical activity in adolescents from public schools: the importance of family and friends. Caderno de Saúde Pública, Rio de Janeiro, v. 30, n. 4, p. 827-838, 2014.

SEABRA, A. F.; MENDONCA, D. M.; THOMIS, M. A.; ANJOS, L. A.; MAIA, J. A. Biological and sociocultural determinants of physical activity in adolescents. Caderno de Saúde Pública, Rio de Janeiro, v. 24, n. 4, p. 721-36, 2008.

SHERAR, L. B.; CUMMING, S. P.; EISENMANN, J. C.; BAXTER-JONES, A. D.; MALINA, R. M. Adolescent biological maturity and physical activity: biology meets behavior. Pediatric Exercise Science, Birmingham, v. 22, n. 3, p. 332-49, 2010.

SOUZA, C. A. D.; RECH, C. R.; SARABIA, T. T.; AÑEZ, C. R. R.; REIS, R. S. Self-efficacy and physical activity in adolescents in Curitiba, Paraná State, Brazil. Caderno de Saúde Pública, Rio de Janeiro, v. 29, n. 10, p. 2039-48, 2013.

SOUZA, O.; SILVA, C.; ASSIS, A. M.; FIACCONE, R. L.; PINTO, J.; MORAES, L. T. Association between physical inactivity and overweight among adolescents in Salvador, Bahia-Brazil. Revista Brasileira de Epidemiologia, Rio de Janeiro, v. 13, n. 3, p. 468-75, 2010. 
TANNER, J. M. Growth at adolescence. 2. ed. Oxford: Blackwell Scientific Publications, 1962.

VEIGA, O. L.; GOMEZ-MARTINEZ, S.; MARTINEZ-GOMEZ, D.; VILLAGRA, A.; CALLE, M. E.; MARCOS, A. Physical activity as a preventive measure against overweight, obesity, infections, allergies and cardiovascular disease risk factors in adolescents: AFINOS Study protocol. BMC Public Health, London, v. 9, n. 475, p. 1-11, 2009.

WHO. Global recommendations on physical activity for health. Geneva: World Health Organization, 2010. Disponível em: <http://www.who.int/dietphysicalactivity/factsheet recommendations/en/>. Acessado em: 01 de fevereiro de 2016.

Autor correspondente: Thiago Silva Piola

E-mail: tspthiago@hotmail.com

Recebido: 09 de outubro de 2017.

Aceito: 26 de abril de 2018. 